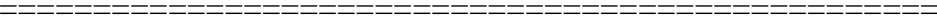

"Supporting Information to be published electronically"

\title{
Mercury bis-Phenyltellurolate as a Precursor for the Synthesis of Binary and Ternary Nanoclusters
}

Davi F. Back, ${ }^{\dagger}$ Gelson N. M. de Oliveira, ${ }^{\dagger}$ Robert A. Burrow, ${ }^{\dagger}$ Ernesto E. Castellano, ${ }^{\dagger}$ Ulrich Abram, ${ }^{\S}$ Ernesto S. Lang ${ }^{*, \dagger}$

\footnotetext{
$\dagger$ Laboratório de Materiais Inorgânicos, Universidade Federal de Santa Maria, 97119-900 Santa Maria, RS, Brazil,

$\$$ Instituto de Física de São Carlos, Departamento de Física e Informática, Universidade de São Paulo, 13560-970, São Carlos, SP, Brazil,

§ Freie Universität Berlin, Institute of Chemistry, Fabeckstr., 34-36, D-14195 Berlin, Germany
}

E-mail address: eslang@quimica.ufsm.br

Tel : 55 (55) 32208980 (Off.) 


\section{Contents}

$\begin{array}{ll}\text { 1. Experimental Section } & \text { Pag. } 3\end{array}$

$\begin{array}{ll}\text { 2. X-ray crystallographic data } & \text { Pag. } 6-7\end{array}$

Crystal data for $\mathbf{1}, \mathbf{2}, \mathbf{3 a}$ and $\mathbf{3 b}$

3. Structure of $\mathbf{1}$ - Figure and selected bond distances $\quad$ Pag. 8

4. Structure of $\mathbf{2}$ - Figure and selected bond distances $\quad$ Pag. 9

5. Structure of 3a- Figure and selected bond distances $\quad$ Pag. 10

6. Structure of $\mathbf{3 b}$ - Figure and selected bond distances $\quad$ Pag. 11

7. Compound 2 showing a pseudo-polymeric structure Pag. 12 


\section{Experimental Section}

Equipments: Elemental analysis were made on a Carlo Erba EA 1110 and EDX(Energy Dispersive X-ray) analysis were made on a LEO mod 440, $20 \mathrm{keV}$ at the Universidade de São Paulo - São Carlos - SP - Brazil.

Typical procedure for 1, $3 \boldsymbol{a}$ and $\mathbf{3 b}$ : A mixture of $\mathrm{Hg}(\mathrm{TePh})_{2}(1.221 \mathrm{~g}, 2.01 \mathrm{mmol})$ and $\left[\mathrm{Ag}\left(\mathrm{PPh}_{3}\right)_{2}\right] \mathrm{NO}_{3}(0.696 \mathrm{~g}, 1.005 \mathrm{mmol})$ for $\mathbf{1}$ and $\left[\mathrm{Co}\left(\mathrm{PPh}_{3}\right)_{2} \mathrm{Cl}_{2}\right](0.338 \mathrm{~g}, 0.516 \mathrm{mmol}$ or $\left[\mathrm{Ni}\left(\mathrm{PPh}_{3}\right)_{2} \mathrm{Cl}_{2}\right](0.338 \mathrm{~g}, 0.516 \mathrm{mmol})$ for $\mathbf{3 a}$ or $\mathbf{3 b}$ respectively, in $15 \mathrm{~mL}$ of dimethylformamide under nitrogen atmosphere were stirred at room temperature for 2 hours.

Compound 1: After a week, stable orange crystals with X-ray-quality were formed. Yield: $0.525 \mathrm{~g}, 42.7 \%$ based on $\mathrm{Hg}(\mathrm{TePh})_{2}$, m.p.: $145-148^{\circ} \mathrm{C}$; Elemental analysis: calc.: $\mathrm{H} 1.64 \%$ C 23.48\%; found: $\mathrm{H} 1.61 \%$, C 23.43\%; Atomic ratios given by EDX analysis: $\mathrm{Hg}_{6} \mathrm{Te}_{16} \mathrm{Ag}_{4}$.

Compound 3a: Prismatic yellow crystals could be isolated from this solution at room temperature after two weeks. Yield: $0.182 \mathrm{~g}, 15.06 \%$ based on $\mathrm{Hg}(\mathrm{TePh})_{2}$, m.p.: $168.5^{\circ}$ C; Elemental analysis: calc.: H 2.11\% C 22.38\% N 1.74\%; found: H 2.14\% C $22.40 \% \mathrm{~N}$ 1.69\%; Atomic ratios given by EDX analysis: $\mathrm{Hg}_{8} \mathrm{Te}_{13} \mathrm{Cl}_{4} \mathrm{Co}_{1}$.

Compound 3b: Prismatic red crystals were isolated from this solution at room temperature after two weeks. Yield: $0.091 \mathrm{~g}, 7.63 \%$ based on $\mathrm{Hg}(\mathrm{TePh})_{2}$, m.p.: $168.5^{\circ}$

C; Elemental analysis: calc.: H $2.11 \%$ C $22.40 \%$ N 1.74\%; found: H $2.07 \%$ C $22.68 \% \mathrm{~N}$ 1.35\%; Atomic ratios given by $\mathrm{EDX}$ analysis: $\mathrm{Hg}_{8} \mathrm{Te}_{13} \mathrm{Cl}_{4} \mathrm{Ni}_{1}$. 
2: A mixture of $\mathrm{Hg}(\mathrm{TePh})_{2}(0.152 \mathrm{~g}, 0.25 \mathrm{mmol})$ and $\left[\mathrm{Ag}\left(\mathrm{PMePh}_{2}\right)_{2}\right] \mathrm{NO}_{3}(0.142 \mathrm{~g}, 0.25$ $\mathrm{mmol}$ ) in $15 \mathrm{~mL}$ of dimethylformamide under nitrogen atmosphere were stirred at 75 $80^{\circ} \mathrm{C}$ for 1 hours. Compound 2: After three days, stable yellow crystals with X-rayquality formed. Yield: $0.044 \mathrm{~g}, 30.5 \%$ based on $\mathrm{Hg}(\mathrm{TePh})_{2}$, m.p.: $\left.145-148^{\circ} \mathrm{C}\right)$; Elemental analysis: calc.: H $1.51 \%$ C $21.81 \%$; found: H $1.53 \%$ C $21.80 \%$. 


\section{X-ray crystallographic data}

Crystallographic measurements were made on a Bruker Kappa Apex II CCD area detector with graphite monochromatized Mo $\mathrm{K} \alpha$ radiation $(\lambda=0.71073 \AA)$. The structure was solved by direct methods (SHELXS-97) and additional atoms were located in the difference Fourier map and refined on $F^{2}$ (SHELXL-97). Metal atoms were refined anisotropically; carbon atoms were refined isotropically; and hydrogen atoms as riding atoms at geometrically defined positions with isotropic thermal parameters $20 \%$ larger than their parent atoms.

Crystal data for 1: $\mathrm{C}_{96} \mathrm{H}_{80} \mathrm{Ag}_{4} \mathrm{Hg}_{6} \mathrm{Te}_{16}$, crystal dimension $=0.20 \times 0.14 \times 0.13 \mathrm{~mm}^{3}$, monoclinic, $C c$ (No. 9), $a=28.961(1) \AA, b=15.597(1) \AA, c=26.911(1) \AA, \beta=$ 113.40(1) $)^{\circ}, V=11156.1(9) \AA^{3}, Z=4, \rho_{\text {calc }}=2.923 \mathrm{mg} / \mathrm{mm}^{3}, 2 \theta_{\max }=26.05^{\circ}$, temperature $=296(2) K$, Mo $\mathrm{K}_{\alpha}(\lambda=0.71073 \AA)$, no. measured reflections $=42102$, unique reflections $=19481\left(R_{\mathrm{int}}=0.0613\right)$. Absorption correction: multi-scan ${ }^{1}: \mathrm{T}_{\min }=$ 0.5318, $\mathrm{T}_{\max }=1.0$. Final residuals $R_{1}=0.0600$ and $w R_{2}=0.1651[I>2 \sigma(I)], 428$ parameters, residual electron density $=3.850 \mathrm{e} \cdot \AA^{-3}$.

Crystal data for 2: $\mathrm{C}_{87} \mathrm{H}_{77} \mathrm{Ag}_{4} \mathrm{Hg}_{6} \mathrm{Te}_{15}$, crystal dimension $=0.15 \times 0.14 \times 0.13 \mathrm{~mm}^{3}$, triclinic, $P-1$ (No. 2), $a=17.571(5) \AA, b=18.633(5) \AA, c=20.902(5) \AA, \alpha=$ $95.805(15)^{\circ}, \beta=113.40(1)^{\circ}, \gamma=90.738(16)^{\circ}, V=6219(3) \AA^{3}, Z=2, \rho_{\text {calc }}=2.511$ $\mathrm{mg} / \mathrm{mm}^{3}, 2 \theta_{\max }=26.05^{\circ}$, temperature $=296(2) K$, Mo $\mathrm{K}_{\alpha}(\lambda=0.71073 \AA)$, no. 
measured reflections $=103025$, unique reflections $=24397\left(R_{\mathrm{int}}=0.1436\right)$. Absorption correction: multi-scan ${ }^{1}: \mathrm{T}_{\min }=0.673, \mathrm{~T}_{\max }=1.00$. Final residuals $R_{1}=0.0746$ and $w R_{2}=$ $0.2041[I>2 \sigma(I)], 423$ parameters, residual electron density $=2.397 \mathrm{e} \cdot \AA^{-3}$.

Crystal data for 3a: $\mathrm{C}_{108} \mathrm{H}_{60} \mathrm{Cl}_{4} \mathrm{CoHg}_{8} \mathrm{~N}_{6} \mathrm{O}_{6} \mathrm{Te}_{13}$, crystal dimension $=0.13 \times 0.12 \mathrm{x}$ $0.11 \mathrm{~mm}^{3}$, cubic, $F-43 c \quad($ No. 219$), a=28.6613(9) \AA, V=23544.4(13) \AA^{3}, Z=8, \rho_{\text {calc }}$ $=2.822 \mathrm{mg} / \mathrm{mm}^{3}, 2 \theta_{\max }=29.61^{\circ}$, temperature $=296(2) K$, Mo $\mathrm{K}_{\alpha}(\lambda=0.71073 \AA)$, no. measured reflections $=19876$, unique reflections $=2757\left(R_{\mathrm{int}}=0.0397\right)$. Absorption correction: multi-scan ${ }^{1}: \mathrm{T}_{\min }=0.653, \mathrm{~T}_{\max }=1.0$. Final residuals $R_{1}=0.0290$ and $w R_{2}=$ $0.0841[I>2 \sigma(I)], 66$ parameters, residual electron density $=1.737$ e. $\AA^{-3}$.

Crystal data for $3 \mathbf{b}: \mathrm{C}_{90} \mathrm{H}_{60} \mathrm{Cl}_{4} \mathrm{NiHg}_{8} \mathrm{~N}_{6} \mathrm{O}_{6} \mathrm{Te}_{13}$, crystal dimension $=0.15 \times 0.14 \mathrm{x}$ $0.12 \mathrm{~mm}^{3}$, cubic, $F-43 c \quad($ No. 219$), a=28.6613(9) \AA, V=23544.4(13) \AA^{3}, Z=8, \rho_{\text {calc }}$ $=2.700 \mathrm{mg} / \mathrm{mm}^{3}, 2 \theta_{\max }=27.98^{\circ}$, temperature $=296(2) K$, Mo $\mathrm{K}_{\alpha}(\lambda=0.71073 \AA)$, no. measured reflections $=83241$, unique reflections $=2386\left(R_{\text {int }}=0.0891\right)$. Absorption correction: multi-scan ${ }^{1}: \mathrm{T}_{\min }=0.490, \mathrm{~T}_{\max }=1.0$. Final residuals $R_{1}=0.0380$ and $w R_{2}=$ $0.1089[I>2 \sigma(I)], 62$ parameters, residual electron density $=1.859 \mathrm{e} . \AA^{-3}$.

(1) R. H. Blessing, Acta Crystallogr., 1995, A51, 33-38. 
Structure of 1

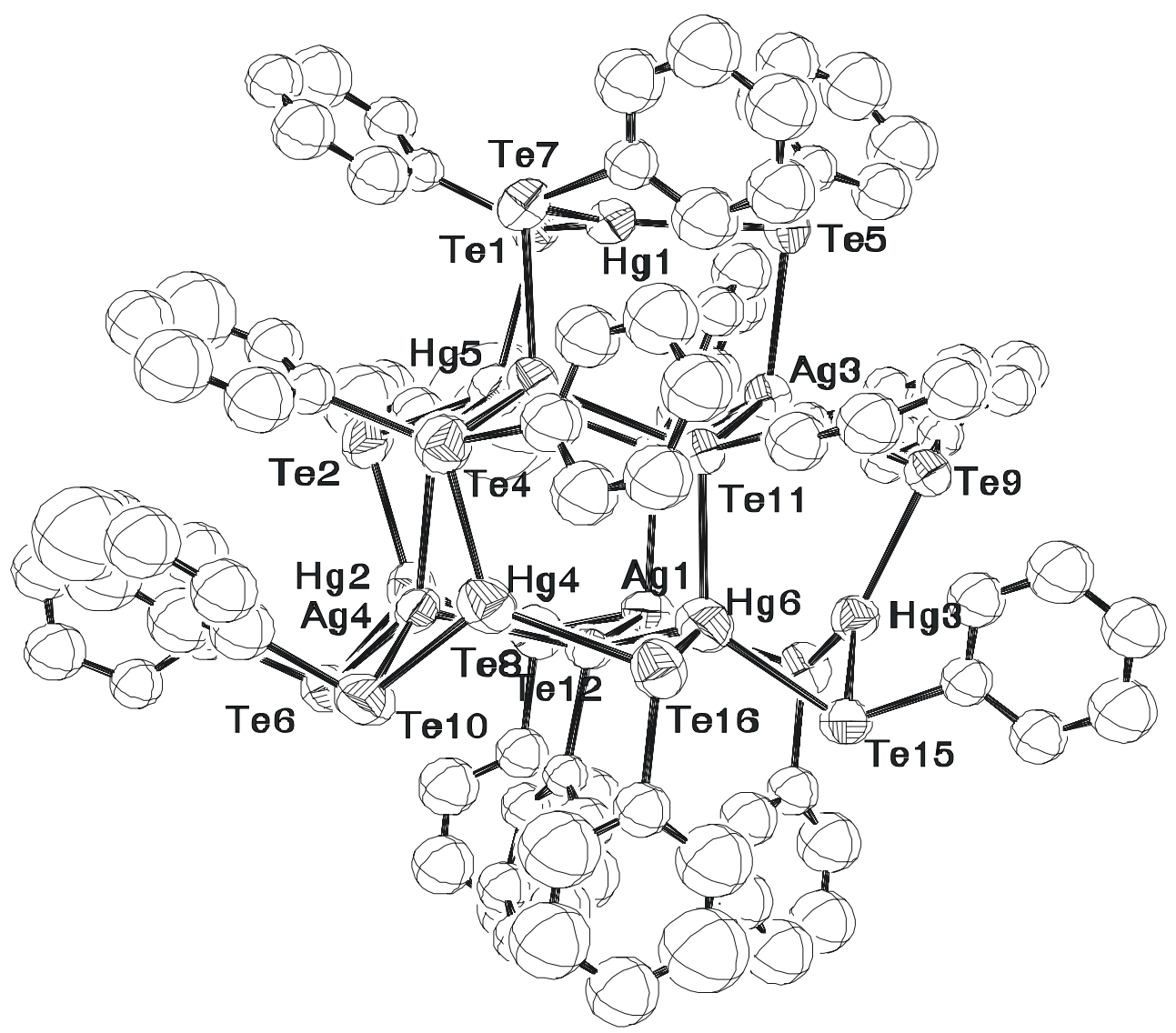

Figure 1. The structure of $\mathbf{1}$ (with $\mathrm{H}$ atoms are omitted and the carbon atoms represented with conventional radius for clarity). Selected bond lengths $[\AA]$ : $\mathrm{Ag}(1)$ $\mathrm{Te}(8)=2.803(2) ; \operatorname{Ag}(1)-\mathrm{Te}(3)=2.8097(18) ; \operatorname{Ag}(1)-\mathrm{Te}(13)=2.839(2) ; \operatorname{Ag}(1)-\mathrm{Te}(12)=$ 2.856(2); $\operatorname{Ag}(2)-\mathrm{Te}(14)=2.812(2) ; \operatorname{Ag}(2)-\mathrm{Te}(4)=2.8166(19) ; \operatorname{Ag}(2)-\mathrm{Te}(7)=2.834(2)$; $\operatorname{Ag}(2)-\operatorname{Te}(11)=2.8373(19) ; \operatorname{Ag}(3)-\operatorname{Te}(3)=2.7818(17) ; \operatorname{Ag}(3)-\operatorname{Te}(11)=2.7969(17) ;$ $\operatorname{Ag}(3)-\operatorname{Te}(5)=2.8269(19) ; \operatorname{Ag}(3)-\operatorname{Te}(9)=2.8574(19) ; \operatorname{Ag}(4)-\operatorname{Te}(4)=2.7524(18) ;$ $\operatorname{Ag}(4)-\mathrm{Te}(12)=2.8135(17) ; \operatorname{Ag}(4)-\mathrm{Te}(6)=2.842(2) ; \operatorname{Ag}(4)-\mathrm{Te}(10)=2.878(2) ; \mathrm{Hg}(1)-$ $\mathrm{Te}(7)=2.6993(17) ; \mathrm{Hg}(1)-\mathrm{Te}(1)=2.7275(16) ; \mathrm{Hg}(1)-\mathrm{Te}(5)=2.7307(15) ; \mathrm{Hg}(2)-$ $\mathrm{Te}(8)=2.702(2) ; \mathrm{Hg}(2)-\mathrm{Te}(6)=2.711(2) ; \mathrm{Hg}(2)-\mathrm{Te}(2)=2.744(2) ; \mathrm{Hg}(3)-\mathrm{Te}(13)=$ 2.6978(16); $\mathrm{Hg}(3)-\mathrm{Te}(9)=2.7115(15) ; \mathrm{Hg}(3)-\mathrm{Te}(15)=2.7402(16) ; \mathrm{Hg}(4)-\mathrm{Te}(10)=$ 2.7017(18); $\mathrm{Hg}(4)-\mathrm{Te}(14)=2.703(2) ; \mathrm{Hg}(4)-\mathrm{Te}(16)=2.7247(19) ; \mathrm{Hg}(5)-\mathrm{Te}(4)=$ 2.7952(17); $\mathrm{Hg}(5)-\mathrm{Te}(3)=2.7994(15) ; \mathrm{Hg}(5)-\mathrm{Te}(2)=2.8080(19) ; \mathrm{Hg}(5)-\mathrm{Te}(1)=$ $2.8289(17) ; \mathrm{Hg}(6)-\mathrm{Te}(11)=2.7713(16) ; \mathrm{Hg}(6)-\mathrm{Te}(12)=2.8168(16) ; \mathrm{Hg}(6)-\mathrm{Te}(15)=$ $2.8307(18) ; \operatorname{Hg}(6)-\mathrm{Te}(16)=2.8390(19)$. 
Structure of 2

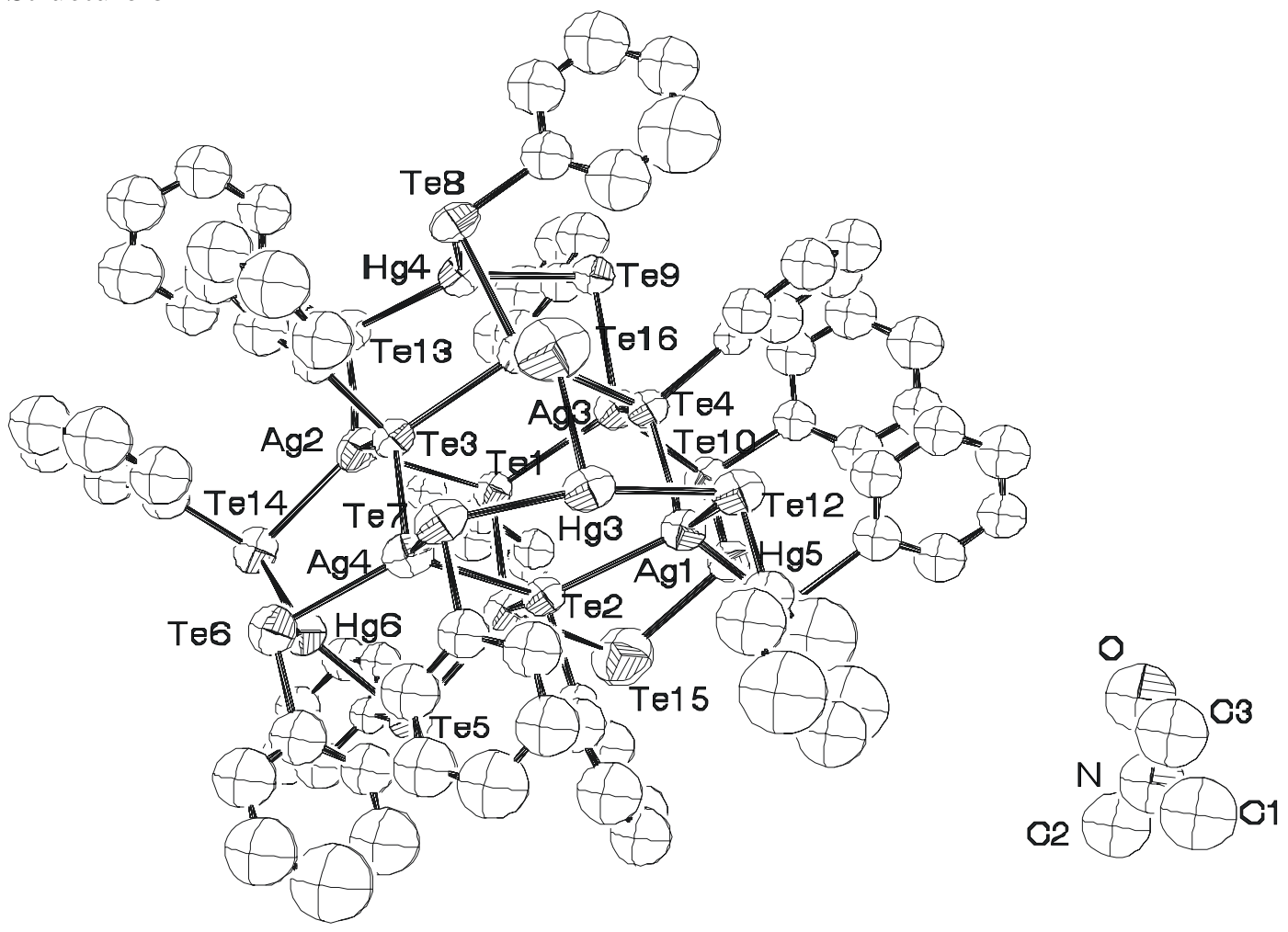

Figure 2. The structure of $\mathbf{2}$ (with $\mathrm{H}$ atoms are omitted and the carbon atoms represented with conventional radius for clarity). Selected bond lengths $[\AA]$ : $\mathrm{Te}(15)-$ $\mathrm{Hg}(2)=2.688(5) ; \mathrm{Te}(15)-\mathrm{Hg}(5)=2.773(6) ; \mathrm{Te}(16)-\mathrm{Hg}(1)=2.694(5) ; \mathrm{Te}(16)-\mathrm{Hg}(3)=$ 2.785(6); $\operatorname{Ag}(1)-\mathrm{Te}(2)=2.779(2) ; \operatorname{Ag}(1)-\mathrm{Te}(4)=2.779(2) ; \operatorname{Ag}(1)-\mathrm{Te}(12)=2.780(2)$; $\operatorname{Ag}(1)-\mathrm{Te}(11)=2.782(2) ; \operatorname{Ag}(2)-\mathrm{Te}(14)=2.781(3) ; \operatorname{Ag}(2)-\mathrm{Te}(13)=2.797(3) ; \operatorname{Ag}(2)-$ $\mathrm{Te}(1)=2.803(2) ; \operatorname{Ag}(2)-\mathrm{Te}(3)=2.819(2) ; \operatorname{Ag}(3)-\mathrm{Te}(9)=2.764(3) ; \operatorname{Ag}(3)-\mathrm{Te}(10)=$ 2.774(2); $\operatorname{Ag}(3)-\mathrm{Te}(4)=2.799(2) ; \operatorname{Ag}(3)-\mathrm{Te}(1)=2.826(2) ; \operatorname{Ag}(4)-\mathrm{Te}(6)=2.762(3)$; $\operatorname{Ag}(4)-\mathrm{Te}(7)=2.771(2) ; \operatorname{Ag}(4)-\mathrm{Te}(3)=2.810(2) ; \operatorname{Ag}(4)-\mathrm{Te}(2)=2.853(2) ; \mathrm{Te}(1)-\mathrm{Hg}(2)$ $=2.7237(19) ; \mathrm{Te}(2)-\mathrm{Hg}(2)=2.7737(17) ; \mathrm{Te}(3)-\mathrm{Hg}(1)=2.7782(19) ; \mathrm{Te}(4)-\mathrm{Hg}(1)=$ 2.7094(17); $\mathrm{Te}(5)-\mathrm{Hg}(2)=2.760(2) ; \mathrm{Te}(5)-\mathrm{Hg}(6)=2.7989(19) ; \mathrm{Te}(6)-\mathrm{Hg}(6)=$ $2.688(2) ; \mathrm{Te}(7)-\mathrm{Hg}(3)=2.630(2) ; \mathrm{Te}(8)-\mathrm{Hg}(4)=2.7662(19) ; \mathrm{Te}(8)-\mathrm{Hg}(1)=$ 2.7775(19); $\mathrm{Te}(9)-\mathrm{Hg}(4)=2.721(2) ; \mathrm{Te}(10)-\mathrm{Hg}(5)=2.640(2) ; \mathrm{Te}(11)-\mathrm{Hg}(5)=$ $2.641(2) ; \mathrm{Te}(12)-\mathrm{C}(121)=2.050(18) ; \mathrm{Te}(12)-\mathrm{Hg}(3)=2.629(2) ; \mathrm{Te}(13)-\mathrm{Hg}(4)=$ 2.690(2); $\mathrm{Te}(14)-\mathrm{Hg}(6)=2.693(2)$. 
Structure of $\mathbf{3 a}$

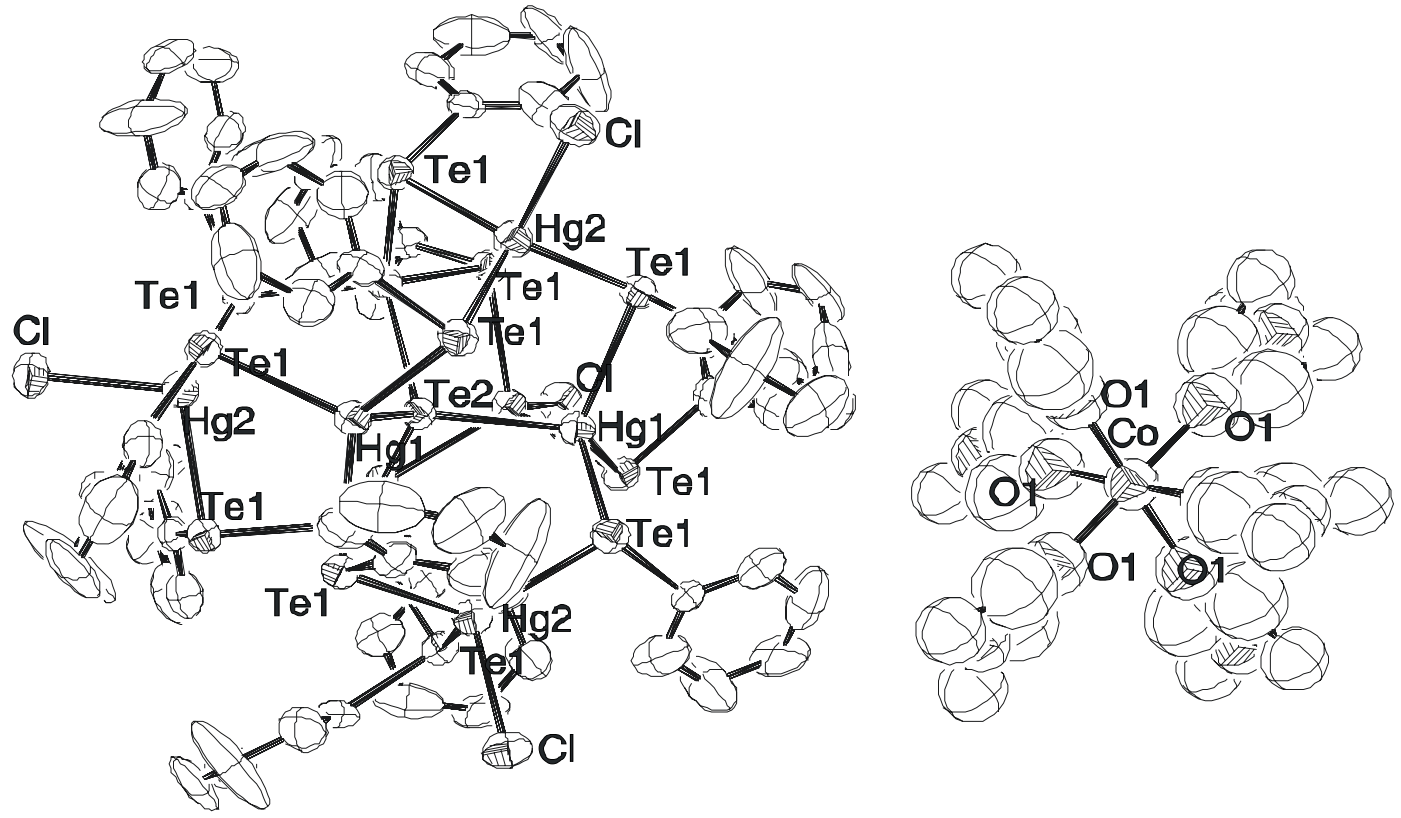

Figure 3a. The structure of 3a (with $\mathrm{H}$ atoms are omitted and the carbon atoms represented with conventional radius for clarity). Selected bond lengths $[\AA \AA]$ and angles $\left[{ }^{\circ}\right]: \operatorname{Te}(1)-\mathrm{Hg}(2)=2.7814(10) ; \mathrm{Te}(1)-\mathrm{Hg}(1)=2.7905(8) ; \mathrm{Te}(2)-\mathrm{Hg}(1) \# 1=2.7649(8)$; $\mathrm{Te}(2)-\mathrm{Hg}(1) \# 2$ = 2.7649(8); $\mathrm{Te}(2)-\mathrm{Hg}(1) \# 3$ = 2.7649(8); $\mathrm{Te}(2)-\mathrm{Hg}(1)=2.7649(8)$; $\mathrm{Hg}(1)-\mathrm{Te}(1) \# 4=2.7905(8) ; \mathrm{Hg}(1)-\mathrm{Te}(1) \# 5=2.7905(8) ; \mathrm{Hg}(2)-\mathrm{Cl}=2.607(6) ; \mathrm{Hg}(2)-$ $\mathrm{Te}(1) \# 6$ = 2.7814(10); Hg(2)-Te(1)\#7 = 2.7814(10); $\mathrm{Hg}(1)-\mathrm{Te}(2)-\mathrm{Hg}(1) \# 1$ = 109.5; $\mathrm{Hg}(1)-\mathrm{Te}(2)-\mathrm{Hg}(1) \# 2=109.5 ; \quad \mathrm{Hg}(1)-\mathrm{Te}(2)-\mathrm{Hg}(1) \# 3=109.5$. Symmetry transformations used to generate equivalent atoms: \#1 = 1-x, 1-y, z; \#2 = x, 1-y, -z; \#3 $=1-\mathrm{x}, \mathrm{y},-\mathrm{z} ; \# 4=\mathrm{y}, \mathrm{x}+0.5, \mathrm{z}-0.5 ; \# 5=\mathrm{y}, 0.5-\mathrm{z}, 0.5-\mathrm{x} ; \# 6=0.5+\mathrm{z}, \mathrm{x},-0.5+\mathrm{y} ; \# 7=1-$ $\mathrm{y}, 0.5+\mathrm{z}, 0.5-\mathrm{x} ; \# 8=0.5+\mathrm{z}, 1-\mathrm{x}, 0.5-\mathrm{y} ; \# 9=0.5-\mathrm{z}, 1-\mathrm{x},-0.5+\mathrm{y} ; \# 10=0.5-\mathrm{z}, \mathrm{x}, 0.5-\mathrm{y} ; \# 11$ $=1-\mathrm{y}, 0.5-\mathrm{z},-0.5+\mathrm{x}$. 

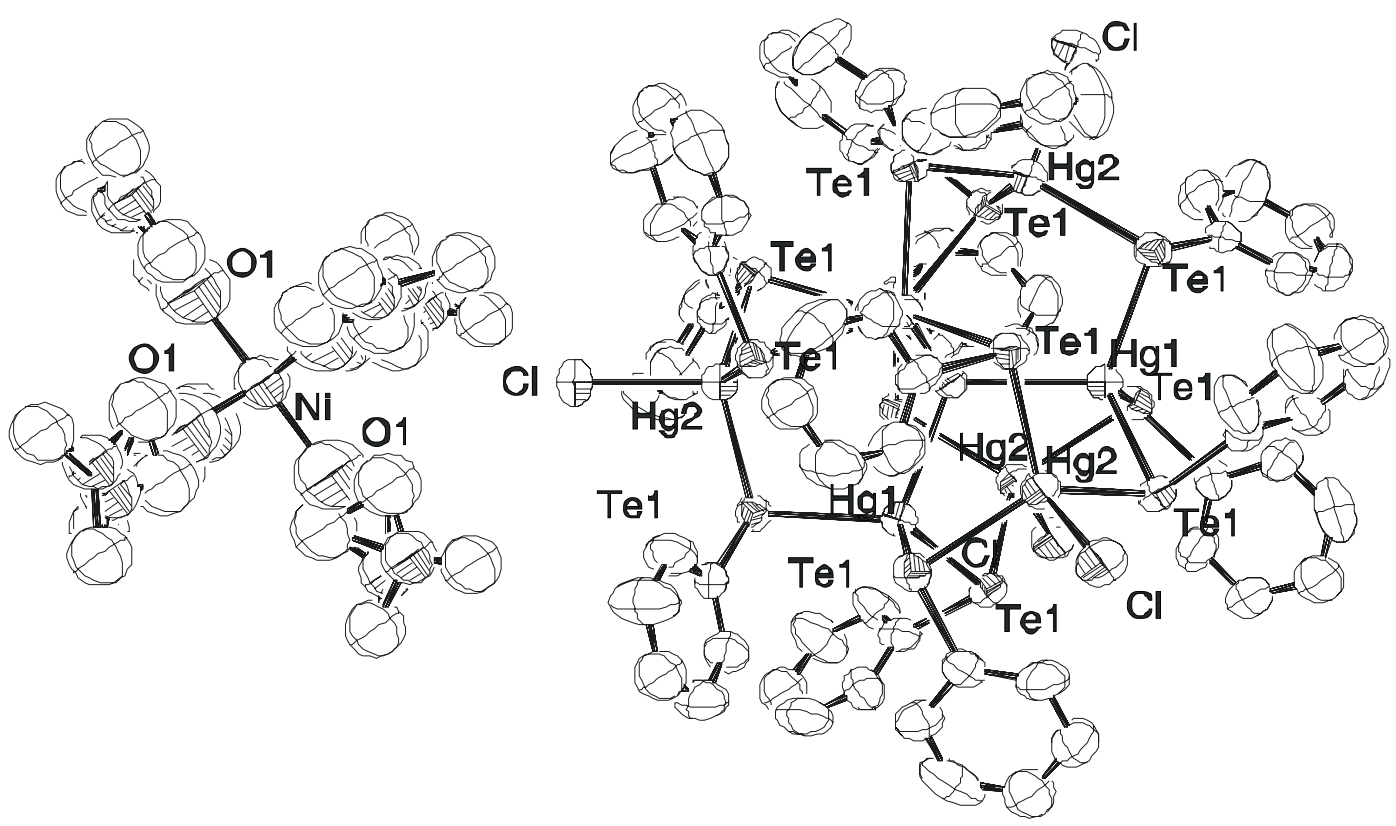

Figure $\mathbf{3 b}$. The structure of $\mathbf{3 b}$ (with $\mathrm{H}$ atoms are omitted and the carbon atoms represented with conventional radius for clarity). Selected bond lengths $[\AA]$ and angles $\left[^{\circ}\right]: \mathrm{Te}(1)-\mathrm{C}(11)=2.112(17) ; \mathrm{Te}(1)-\mathrm{Hg}(2)=2.775(2) ; \mathrm{Te}(1)-\operatorname{Hg}(1)=2.7914(18)$; $\mathrm{Te}(2)-\mathrm{Hg}(1) \# 1$ = 2.7735(17); $\mathrm{Te}(2)-\mathrm{Hg}(1)=2.7735(17) ; \mathrm{Te}(2)-\mathrm{Hg}(1) \# 2=2.7735(17)$; $\mathrm{Te}(2)-\mathrm{Hg}(1) \# 3=2.7735(17) ; \quad \mathrm{Hg}(1)-\mathrm{Te}(1) \# 4=2.7914(18) ; \quad \mathrm{Hg}(1)-\mathrm{Te}(1) \# 5=$ 2.7914(18); $\mathrm{Hg}(2)-\mathrm{Cl}=2.613(13) ; \mathrm{Hg}(2)-\mathrm{Te}(1) \# 6=2.775(2) ; \mathrm{Hg}(2)-\mathrm{Te}(1) \# 7=$ 2.775(2); $\mathrm{Ni}-\mathrm{O}(1)=2.1575 ; \mathrm{Hg}(2)-\mathrm{Te}(1)-\mathrm{Hg}(1)=96.76(6) ; \mathrm{Hg}(1) \# 1-\mathrm{Te}(2)-\mathrm{Hg}(1)=$ 109.5; $\mathrm{Hg}(1) \# 1-\mathrm{Te}(2)-\mathrm{Hg}(1) \# 2=109.5 ; \mathrm{Hg}(1)-\mathrm{Te}(2)-\mathrm{Hg}(1) \# 2=109.5 ; \mathrm{Hg}(1) \# 1-\mathrm{Te}(2)-$ $\mathrm{Hg}(1) \# 3=109.5 ; \mathrm{Hg}(1)-\mathrm{Te}(2)-\mathrm{Hg}(1) \# 3=109.5 ; \mathrm{Hg}(1) \# 2-\mathrm{Te}(2)-\mathrm{Hg}(1) \# 3=109.5 ;$ $\mathrm{Te}(2)-\mathrm{Hg}(1)-\mathrm{Te}(1)=\quad$ 106.46(5); $\mathrm{Te}(2)-\mathrm{Hg}(1)-\mathrm{Te}(1) \# 4=106.46(5) ; \mathrm{Te}(1)-\mathrm{Hg}(1)-$ $\mathrm{Te}(1) \# 4=112.31(5) ; \quad \mathrm{Te}(2)-\mathrm{Hg}(1)-\mathrm{Te}(1) \# 5 \quad=106.46(5) ; \mathrm{Te}(1)-\mathrm{Hg}(1)-\mathrm{Te}(1) \# 5=$ 112.31(5); Te(1)\#4-Hg(1)-Te(1)\#5 = 112.31(5); Te(1)\#6-Hg(2)-Te(1)\#7 = 115.18(3); $\mathrm{Te}(1) \# 6-\mathrm{Hg}(2)-\mathrm{Te}(1)=115.18(3)$. 
Compound 2 showing a pseudo-polymeric structure

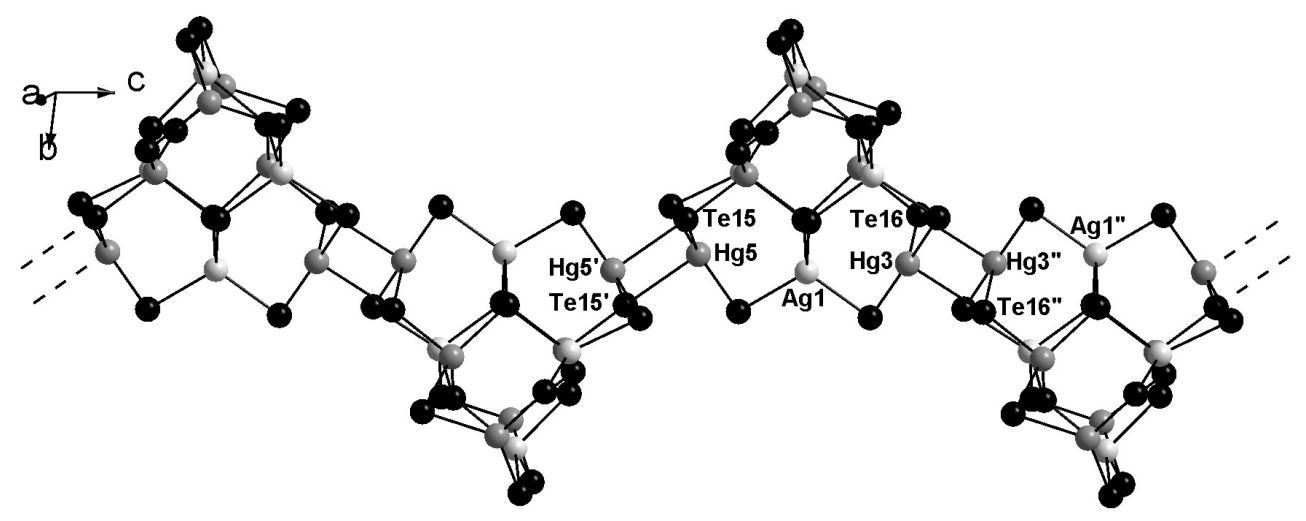

Figure 4. The structure of 2 with disordered $\mu_{2}$-Te atom appearing in two positions (Te15 and Te16) with a occupancy of 0.5 for each tellurium atom showing a pseudopolymeric structure in direction of the crystallographic axis $c$; phenyl rings are omitted for clarity. Selected bond lengths $[\AA]]$ : $\mathrm{Te}(15)-\mathrm{Hg}(5)^{\prime}=3.144(5) ; \mathrm{Te}(16)-\mathrm{Hg}(3)$ ' " = 3.181(5); $\operatorname{Hg}(3)-\mathrm{Te}(16){ }^{\prime}=3.181(5) ; \operatorname{Hg}(5)-\mathrm{Te}(15){ }^{\prime}=3.144(5)$. Symmetry transformations used to generate equivalent atoms: (') $=-x+2,-y+1,-z+2$; (") $=-x+2,-$ $\mathrm{y}+1,-\mathrm{z}+3$. 